\title{
Article \\ CGRP Inhibitors and Oxidative Stress Biomarkers in Resistant Migraine: A Real-Life Study with Erenumab, Fremanezumab, and Galcanezumab
}

\author{
Ciro De Luca ${ }^{1,2}$ (D), Filippo Baldacci ${ }^{2}$ *(D), Sonia Mazzucchi ${ }^{2}$, Irene Lombardo ${ }^{2}$, Letizia Curto ${ }^{2}$, Martina Ulivi ${ }^{2}$, \\ Lucia Chico $^{2}$, Michele Papa ${ }^{1,3} \mathbb{D}$, Gabriele Siciliano ${ }^{2}$ and Sara Gori ${ }^{2}$
}

check for updates

Citation: De Luca, C.; Baldacci, F.; Mazzucchi, S.; Lombardo, I.; Curto, L.; Ulivi, M.; Chico, L.; Papa, M.; Siciliano, G.; Gori, S. CGRP Inhibitors and Oxidative Stress Biomarkers in Resistant Migraine: A Real-Life Study with Erenumab, Fremanezumab, and Galcanezumab. J. Clin. Med. 2021, 10, 4586. https://doi.org/10.3390/ jcm10194586

Academic Editor: Stefan Evers

Received: 31 August 2021

Accepted: 1 October 2021

Published: 4 October 2021

Publisher's Note: MDPI stays neutral with regard to jurisdictional claims in published maps and institutional affiliations.

Copyright: (c) 2021 by the authors. Licensee MDPI, Basel, Switzerland. This article is an open access article distributed under the terms and conditions of the Creative Commons Attribution (CC BY) license (https:/ / creativecommons.org/licenses/by/ $4.0 /)$.
1 Laboratory of Morphology of Neuronal Network, Department of Public Medicine, University of Campania “Luigi Vanvitelli", 80138 Napoli, Italy; ciro.deluca@unicampania.it (C.D.L.); michele.papa@unicampania.it (M.P.)

2 Neurology Unit, Department of Clinical and Experimental Medicine, University of Pisa, 56126 Pisa, Italy; mazzucchi.s@gmail.com (S.M.); irene.lombardo@hotmail.com (I.L.); letiziacurto@hotmail.it (L.C.); martinaulivi@gmail.com (M.U.); 1.chico@ao-pisa.toscana.it (L.C.); gabriele.siciliano@unipi.it (G.S.); sara-gori@libero.it (S.G.)

3 SYSBIO Centre of Systems Biology ISBE.ITALY, University of Milano-Bicocca, 20126 Milano, Italy

* Correspondence: filippo.baldacci@unipi.it

\begin{abstract}
Patients with high-frequency resistant migraine and medication-overuse headache are still the main clinical challenge in tertiary headache centers. The approval of targeted antibodies against the calcitonin gene-related peptide (CGRP) and its receptor represents a powerful instrument. In this study, we observed how biological and clinical features of resistant migraineurs responded to erenumab, fremanezumab, or galcanezumab. We found a reduction in advanced oxidation protein products (AOPP) as a biomarker of improved redox state after six months of treatment. We also found that treatment efficacy was precocious and maintained with high individual responder rates. In particular, seven out of ten patients achieved a reduction of $50 \%$ from the baseline at three months, which was maintained at six months, while about one out of our patients experienced a $75 \%$ reduction in headache frequency from the first month of treatment. The migraine disability assessment (MIDAS) and the associated fatigue, anxiety, and sleep quality also significantly improved. The allodynia symptom dropped from moderate/severe to mild/absent as a sign of central sensitization reduction. Our study confirmed the safety and efficacy of CGRP inhibition in real-life, high-challenging patients. Additional evidence is needed to understand the role of oxidative stress as a migraine biomarker.
\end{abstract}

Keywords: resistant migraine; CGRP; oxidative stress; biomarkers; medication-overuse headache

\section{Introduction}

Migraine with or without an aura is one of the most frequent and disabling neurological diseases [1,2]. Migraine affects young, prevalently female, patients during their most productive and socially active years of life. The migraine disease expresses highly variable phenotypes considering just the frequency and intensity of the attacks. The pain phase of migraine is usually treated with non-steroidal anti-inflammatory drugs (NSAIDs), triptans, or a combination of analgesics. Prophylactic drugs are also available for patients with more than four days/month of headache to reduce the frequency of the attacks. All these drugs were used for other conditions (e.g., depression, epilepsy, hypertension, spasticity) and found to be effective for migraine treatment [3]. In the last few years, the approval of targeted drugs represented a paradigm shift for migraine, as these molecules designed to block the signaling of the calcitonin gene-related peptide (CGRP), which is thought to be the main culprit for migraine pathogenesis $[4,5]$.

The clinical challenge is still harsh, especially for the chronic migraineurs, which suffer more than 15 days/month of headache and in many cases present both prophylactic 
failure (resistant or even refractory migraine) and acute medication overuse [6]. The lack of personalized therapy is one of the unmet needs of migraine. Even though comorbidities usually guide the clinician to prefer a prophylactic or acute medication over another, there is still no possibility to predict drug efficacy before its assumption. The trial and error prescriptions that the patient experiences, even when correctly informed by the specialist, are psychologically detrimental [7].

A novel biomarker could both shed some light on the migraine pathophysiology and lead towards precision-medicine targets [8-11].

Hemodynamic changes related to neurotransmitters concentration, physical ac-tivity, and the associated oxidative stress are important fields of investigation in mi-graine to achieve this goal $[12,13]$.

We previously proposed that the oxidative mitochondrial metabolism could play a role, particularly in chronic migraine with central sensitization [14]. The clinically accessible dosage of plasmatic levels of advanced oxidation protein products (AOPP), ferric-reducing antioxidant power (FRAP), and thiolic groups (-SH) are affordable, though indirect, measurements of the patient's redox state. They turned out to be significantly different between chronic migraine patients and healthy controls and positively modulated after OnabotulintoxinA (BoNT/A) treatment [14,15]. The exact mechanisms of BoNT/A effectiveness in chronic migraine prophylaxis are not clear; however, lower peripheral plasmatic levels of CGRP have been reported after BoNT/A treatment [16]. However, aside from nociception, CGRP plays a role in oxidative stress, as reported by recent studies [17-19].

The objectives of this study were: (i) to examine the levels of the plasmatic oxidative stress biomarkers AOPP, FRAP, and -SH in resistant migraineurs after six months of treatment with monoclonal antibodies against CGRP or its receptor and (ii) to evaluate possible variations of migraine clinical features and comorbidities through validated scales.

\section{Materials and Methods}

\subsection{Study Population}

We carried out a prospective study recruiting consecutive outpatients of the Headache Centre of the University of Pisa, from July 2020 to June 2021, with the enlisted inclusion (IC) and exclusion criteria (EC). IC: (1) adult patient, age $\geq 18$ years; (2) patient fulfilling the International Classification of Headache Disorders-3 (ICHD-3) criteria for episodic migraine without or with aura or chronic migraine, and medication-overuse headache (ICHD-3 1.1 or 1.2 or 1.3, and 8.2) [1]; (3) frequency of headache between 8 and 14 days/month for patients with episodic migraine (ICHD-3 1.1 or 1.2); (4) patient fulfilling the European Headache Federation (EHF) consensus on the definition of resistant migraine [20]; (5) absence of migraine preventive treatment for at least 3 months before study inclusion; (6) eligibility for erenumab, fremanezumab, and galcanezumab. EC: (1) comorbid medical disorders and treatments for chronic systemic diseases; (2) pregnancy or breastfeeding during the study.

All patients were not enrolled in previous clinical studies on migraine and were not previously treated with monoclonal antibodies against CGRP. This study was performed in accordance with the Declaration of Helsinki, and it was approved by the local ethics committee (Comitato Etico Area Vasta Nord Ovest-Sezione Autonoma del Comitato Etico Regionale per la Sperimentazione Clinica—Via Roma 67, 56126, Pisa, Italy) with approval code ID_14518. All subjects involved provided written, informed consent before their inclusion.

\subsection{Clinical Assessment}

Patients underwent four visits: baseline assessment (T0) and 1-month (T1), 3-months (T2), and 6-months (T3) follow-up visits. At the end of each visit (T0, T1, T2, and T3), a blood sample was collected for biochemical analysis. Each visit was performed before the administration of the drug. To resolve the different periodicity of the treatment, a standard month of 30 days was considered with a -4 days window to schedule the next visit of patients receiving galcanezumab and fremanezumab and a -2 days window for patients treated with erenumab. 
Furthermore, clinical features of migraine were measured through an interview and patients' self-reported diary as well as the following scales: Migraine Disability Assessment (MIDAS) for the assessment of migraine-related disability (T0, T2, T3), Allodynia Symptoms Checklist 12 (ASC-12) for a specific measure of ictal allodynia (T0, T3), the Fatigue Severity Scale (FSS) to evaluate migraine-associated fatigue (T0, T3), and the Generalized Anxiety disorder (GAD-7) and Patient Health Questionnaire (PHQ-9), to evaluate anxiety and symptoms of mood disorders (T0, T3). Sleep quality was determined (T0, T3) through the Pittsburgh Sleep Quality Index (PSQI). The migraine frequency was evaluated on a single month for T1 and over the previous two months and three months for T2 and T3, respectively.

\subsection{Biochemical Analysis}

Blood samples were collected during the T0, T1, T2 and T3 visits. All the venous samples were collected early in the morning (around 9 a.m.), after a light breakfast. Blood sample vials were centrifuged within $2 \mathrm{~h}$ from the collection $\left(2500 \mathrm{~g}\right.$ for $10 \mathrm{~min}-4{ }^{\circ} \mathrm{C}$ ) for plasma separation and processed afterward or stored at $-80^{\circ} \mathrm{C}$ and analyzed within 1 month. Plasmatic levels of AOPP, FRAP, and -SH were measured as previously described [14]. The biochemical analysis was performed at the laboratory of the Neurology Unit.

AOPP are indicators of oxidative damage, particularly to proteins, including bioproducts of the myeloperoxidase enzyme reacting with plasma proteins. Data were shown as $\mathrm{nmol} / \mathrm{mL}$ of chloramine equivalents (CE).

The FRAP measurement estimates the non-enzymatic antioxidant properties of plasma. The data were expressed as $\mathrm{mmol} / \mathrm{L}$.

The measure of sulfhydryl groups (-SH), also known as thiol groups, expressed the property shared by some plasma molecules, such as glutathione (GSH), to oppose the propagation of oxidative processes and enzymatically revert the oxidation. Data were expressed as $\mathrm{mol} / \mathrm{L}$.

\subsection{Statistical Analysis}

Quantitative variables were not normally distributed (Shapiro-Wilk test). The continuous variables were expressed as medians and interquartile ranges (IQR). The categorical variables were expressed as frequency percentages. The comparison between non-parametric quantitative variables was performed employing the Wilcoxon test to compare the difference at $\mathrm{T} 0, \mathrm{~T} 1, \mathrm{~T} 2$, and $\mathrm{T} 3$ of the quantitative variables within the migraine population. The statistical significance threshold was set at $p>0.05$. A correction for multiple comparisons with Bonferroni was applied for biochemical analysis $(p=0.016)$. The comparison between categorical dichotomous variables was performed by the chi-square test with continuity correction (Yates or Fisher exact test when necessary). SPSS version 24.0 for Windows was used for statistical analyses

\section{Results}

The patients enrolled in the study were 42 , of whom $33(78.6 \%)$ were females, and $9(21.4 \%)$ were males; their ages comprised between 27 and 74 years old, with a median of 52 (14.75). All the patients were diagnosed, according to the ICHD-3 as migraine without aura, and 4 patients $(9.5 \%)$ also received the migraine-with-aura diagnosis. High-frequency episodic migraine was assessed in 6 patients $(14.3 \%)$, while the remaining $36(85.7 \%)$ were diagnosed with chronic migraine. All chronc migraine patients fulfilled the criteria for medication-overuse headache $(\mathrm{MOH})$.

The years of illness were between 10 and 57 years with a median of 32.5 (IQR 14.5).

The patients were treated as follows: 23 received erenumab $70 \mathrm{mg}$; galcanezumab was prescribed to 17 patients ( $240 \mathrm{mg}$ loading dose, $120 \mathrm{mg}$ monthly); 2 patients were enrolled with fremanezumab $225 \mathrm{mg}$. 
Two patients experienced slight side effects (pain at the site of injection), one with erenumab and one with galcanezumab, while none discontinued the prescribed drug.

All our patients were resistant to four of the recommended classes of prophylactic drugs considering antiepileptics, tricyclic antidepressants, beta-blockers, angiotensinreceptor blockers (ARBs), and calcium-channel blockers. Two-thirds (30 patients) were also resistant to selective serotonin reuptake inhibitors (SSRIs) or serotonin-norepinephrine reuptake inhibitors (SNRIs). Eventually, 25 patients underwent at least 3 cycles of BoNT/A administration.

The headache frequency (Table 1), expressed in days/month, for all the patients underwent a significant decrease considering the T0-T1 comparison (T0: 22.0, IQR 14; T1: 12.0, IQR 12; $p<0.001)$.

Table 1. Clinical features comparison at different time points. Values are expressed as median (IQR) and the number of patients $(n)$.

\begin{tabular}{cccccc}
\hline & T0 & T1 & T2 & T3 & $p$ (Wilcoxon Test) \\
\hline Frequency & $22.0(14)$ & $12.0(12)$ & $8.0(11.5)$ & $9.0(8)$ & T0-T1: $p<0.001^{* * *}$ \\
(days/month) & $n=42$ & $n=41$ & $n=33$ & $n=22$ & T0-T2: $p<0.001^{* * *}$ \\
& $81(69)$ & & $23.5(31.25)$ & $14(17.75)$ & T0-T3: $p=0.003^{* *}$ \\
MIDAS $^{*}$ & $n=42$ & - & $n=32$ & $n=22$ & T0-T3: $p<0.001^{* * *}$ \\
& $45(18)$ & & & $27(20.25)$ & $p=0.038^{*}$ \\
FSS $^{*}$ & $n=39$ & & $n=20$ & \\
& $7(5)$ & & $2(3)$ & $p=0.001^{* *}$ \\
ASC-12 $^{3}$ & $n=39$ & & $n=22$ & \\
& $9(9)$ & & $5(2.5)$ & $p=0.002^{* *}$ \\
GAD-7 $^{4}$ & $n=39$ & & $n=21$ & \\
PHQ-9 $^{5}$ & $8(8)$ & & & $n .5(7)$ & \\
& $n=39$ & & & $n=22$ & \\
PSQ I $^{6}$ & $11(11)$ & & & $n=0.393$
\end{tabular}

${ }^{1}$ Migraine Disability Assessment Scale; ${ }^{2}$ Fatigue Severity Scale; ${ }^{3}$ Allodynia Symptom Checklist $12 ;{ }^{4}$ Generalized Anxiety Disorder 7; ${ }^{5}$ Patient Health Questionnaire 9; ${ }^{6}$ Pittsburgh Sleep Quality Index; ${ }^{* * *} p<0.001 ;{ }^{* *} p<0.01$; $* p<0.05)$.

The analysis of the T2 and T3 time points revealed a stable improvement in the headache frequency. In particular, for the 33 patients, we observed at T2 (3 months from the baseline) a significantly lower median of 8 days/month (IQR 11.5), compared to the T0 $(p<0.001)$. The same can be stated about the 22 patients at T3 $(9.0$, IQR $8, p=0.003)$.

After three months of treatment, $61.3 \%$ of patients (19 out of 31) initially diagnosed with $\mathrm{MOH}$ and chronic migraine suspended the overuse. The result was even more evident at $\mathrm{T} 2$ with $70 \%$ of patients (14 out of 20 ) that achieved $\mathrm{MOH}$ cessation.

The MIDAS score (Table 1) showed an improvement in the quality of life with the reduction in the migraine-related disability $(81, \mathrm{IQR} 69)$ both at T2 $(23.5$, IQR $31.25 ; p<0.001)$ and T3 (14, IQR 17.75; $p<0.001)$.

Another endpoint we considered was the individual responder rate, for reduction in the headache frequency higher than $50 \%$ or $75 \%$ compared to the baseline.

Compared to T0, $51.2 \%$ of patients achieved a $50 \%$ reduction in their headache frequency after 1 month of treatment, while $69.7 \%$ achieved this endpoint at T2, and approximately the same percentage maintained it at 6 months $(68.2 \%)$.

Moreover, a reduction of $75 \%$ in the baseline headache frequency was observed consistently at all time points, specifically: $24.4 \%$ of patients after 1 month, $36.4 \%$ at T2, and $27.3 \%$ at $\mathrm{T} 3$.

Allodynia was significantly reduced as indicated by the ASC-12 evaluation (Table 1, Figure 1), which showed a median value of 7 (IQR 5) at T0 and which dropped to 2 (IQR 3; $p=0.001)$ at $\mathrm{T} 3$. 


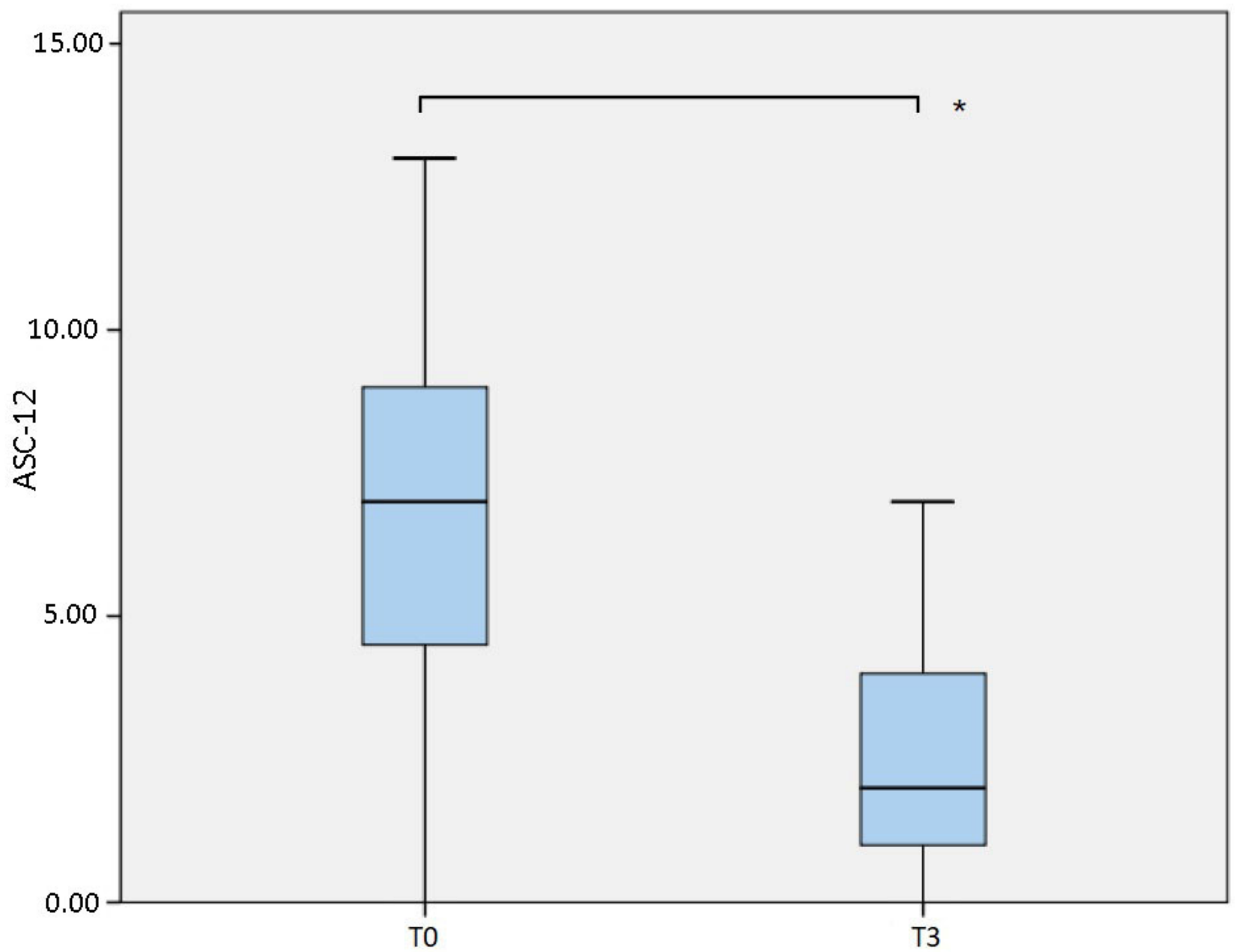

Figure 1. Comparison of Allodynia Symptoms Checklist 12 (ASC-12) measured at baseline (T0) and after 6 months (T3). ${ }^{*} p=0.001$.

Furthermore, the associated fatigue was significantly reduced comparing T0 and T3 (T0: 45, IQR 18; T3: 27, IQR 20.25; $p=0.038$ ).

Considering the anxiety symptoms, the GAD-7 score indicated a significant decrease from a median of 9 (IQR 9) at T0 to 5 (IQR 2.5) after 6 months of therapy $(p=0.002)$, while the mood impairment, assessed through the PHQ-9, showed no significant differences (Table 1).

The PSQI demonstrated a significant reduction from a median of 11 (IQR 11) to 7 (IQR 6.5) comparing the baseline to T3 $(p=0.004)$; however, it did not reach the cutoff of $<5$ for a good quality of sleep (Table 1).

A significant reduction in the advanced oxidation products AOPP (Table 2, Figure 2) was measured $(p=0.004)$, comparing the samples obtained at T0 (median $291.4 \mathrm{nmol} / \mathrm{L}$, IQR 154.25) and T3 (median $236.2 \mathrm{nmol} / \mathrm{L}$, IQR 111.2). The result was obtained considering 37 patients at baseline and 11 patients at T3. If the comparison is conducted considering the same 11 patients at both T0 (median $336.0 \mathrm{nmol} / \mathrm{L}, \mathrm{IQR} 91.3$ ) and T3, the difference is even more evident $(p=0.004)$. 
Table 2. Biochemical analysis of redox properties of patients' plasma at different time points. Values are expressed as median (IQR) and the number of patients $(n)$.

\begin{tabular}{cccccc}
\hline & T0 & T1 & T2 & T3 & $p$ (Wilcoxon Test) \\
\hline & $291.4(154.25)$ & $263.2(139.5)$ & $299.0(166.3)$ & $236.2(111.2)$ & T0-T1: $p=0.306$ \\
AOPP $^{1}$ & $n=37$ & $n=19$ & $n=15$ & $n=11$ & T0-T2: $p=0.272$ \\
& & & & T0-T3: $p=0.004^{*}$ \\
& & & & & T0-T1: $p=0.085$ \\
FRAP $^{2}$ & $0.603(0.0815)$ & $0.616(0.062)$ & $0.561(0.071)$ & $0.584(0.061)$ & T0-T2: $p=0.041$ \\
& $n=37$ & $n=19$ & $n=15$ & $n=11$ & T0-T3: $p=0.859$ \\
& & & & & T0-T1: $p=0.603$ \\
-SH $^{3}$ & $0.235(0.081)$ & $0.271(0.062)$ & $0.276(0.071)$ & $0.272(0.061)$ & T0-T2: $p=0.334$ \\
& $n=37$ & $n=19$ & $n=15$ & $n=11$ & T0-T3: $p=0.110$
\end{tabular}

${ }^{1}$ Advanced Oxidation Protein Products; ${ }^{2}$ Ferric-Reducing Antioxidant Power; ${ }^{3}$ Thiolic Groups; $\left({ }^{*} p<0.01\right)$.

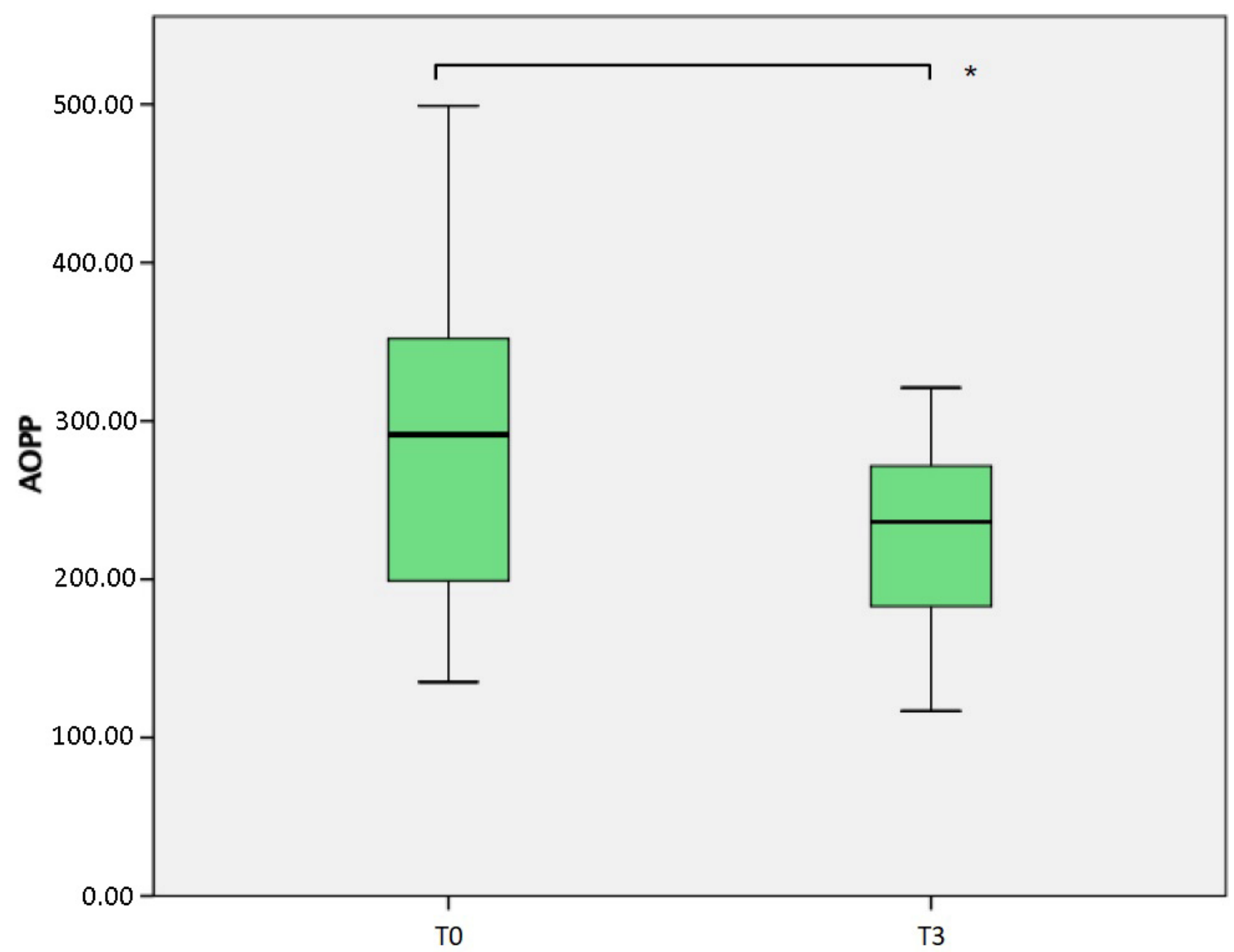

Figure 2. Comparison of Advanced Oxidation Protein Products (AOPP) measured at baseline (T0) and after 6 months (T3). ${ }^{*} p=0.004$.

The values indicating the antioxidant power of the plasma showed no significant differences with the baseline and both the FRAP and -SH (Table 2). The latter exhibited an increasing trend at $\mathrm{T} 1$ (median $0.271 \mu \mathrm{mol} / \mathrm{L}, \mathrm{IQR} 0.062)$, T2 $(0.276 \mu \mathrm{mol} / \mathrm{L}, \mathrm{IQR} 0.071)$, and T3 (median $0.272 \mu \mathrm{mol} / \mathrm{L}$, IQR 0.061) compared to T0 (median $0.235 \mu \mathrm{mol} / \mathrm{L}$, IQR 0.0815), however without reaching statistical significance. 


\section{Discussion}

The population of the study was selected to express a severe phenotype of migraine (either high-frequency episodic or chronic migraine), with all chronic migraine patients being diagnosed with $\mathrm{MOH}$ and all patients fulfilling the EHF definition of resistant migraine.

Nonetheless, the CGRP system inhibition (erenumab, fremanezumab, galcanezumab) managed to improve the clinical features of migraine. Particularly significant was the related disability measured with MIDAS that, at baseline, had a median value of 81 , which was far above the cutoff of 21 for "severe disability" and which dropped to a median value of $14(p<0.001)$ at six months in the range of "moderate disability." The reduction in the MIDAS score was evident also after three months, even remaining in the severe disability range (median value $=23.5$ ).

The treatments improved migraine symptoms, particularly allodynia, which ameliorated from moderate/severe to mild/absent after six months $(p=0.001)$. Considering allodynia as a measure of central sensitization, this result can be interpreted as the positive interference of the therapy on the trigeminovascular system [21] either directly or as a consequence of the clinical improvement. In chronic migraine, the prolonged painful stimulation could induce sensitization, particularly in the caudal trigeminal nucleus and in the trigeminothalamic cortical circuitry, clinically manifesting as allodynia [22].

The clinical improvement was indeed remarkable, with more than half of the patients achieving an individual reduction of $50 \%$ in headache frequency after one month, and with almost 7 out of 10 patients experiencing the same improvement after three and six months. The result was coupled with a $61.3 \%$ and $70 \%$ reduction in patients with a $\mathrm{MOH}$ diagnosis at $\mathrm{T} 2$ and $\mathrm{T} 3$, respectively.

Furthermore, a significant percentage of the patients $(24.4 \%$ at $\mathrm{T} 1,36.4 \%$ at $\mathrm{T} 2$, and $27.3 \%$ at T3) reported an individual reduction of $75 \%$ in their headache frequency.

These data are corroborating the efficacy of the anti-CGRP treatment even in a severe migraine phenotype.

The associated fatigue, measured through the FSS, consistently showed a significant reduction, as well as the anxiety symptoms (GAD-7), demonstrating an overall improvement in the associated comorbidities, together with the migraine disease itself. The amelioration of sleep quality, on the other hand, even if statistically significant was not enough to reach a good quality of sleep.

This parameter is important, as recently demonstrated during the pandemic when both physical activity levels and sleep quality dropped in migraine patients [23]. The link between oxidative stress, physical activity, and sleep quality could somehow reside in the CGRP system $[12,13,23]$.

The safety of the antibodies' anti-CGRP, or the associated receptor, was confirmed in this real-life study, considering the minor side effects that never led to drug discontinuation.

The results of this study are in line with previous observations of increased oxidative stress in migraine patients $[14,15,17,24,25]$. The oxidative state of plasma proteins and the antioxidant reservoir were measured through the biomarkers AOPP, FRAP, and -SH. In our previous investigation, we found that chronic migraine with medication overuse reduced the antioxidant power of plasma [14]. This evidence was subsequently confirmed, together with the increase in AOPP, as a marker of advanced oxidation [15]. Moreover, in the aforementioned study, we investigated the role of BoNT/A considering both clinical and oxidative stress parameters.

We hypothesized that the complex action of the toxin, among other features, chronically blocked the release of CGRP, reducing peripheral sensitization secondary to "neurogenic" inflammation and consequently the oxidative stress [15]. The direct interference with the CGRP system aids the validation of our previous experimental data and adds more information about migraine pathophysiology biomarkers.

We confirmed a significant reduction in AOPP $(p=0.004)$ with chronic inhibition of the CGRP system, after six months of treatment. We did not demonstrate an increase in FRAP and -SH, as previously reported after BoNT/A treatment. This study suggests that 
the increase in antioxidant power of the patients' plasma could be unrelated to the disease severity and at least partly dependent on the chosen treatment.

Studying the biomarkers, the tonic blockage of CGRP signaling did not affect the reduction in the oxidation products, which was also observed with BoNT/A treatment [15]. However, we failed to demonstrate the increase in the antioxidant power of plasma, found with the BoNT/A therapy, either for the different mechanism of action or for the small population size.

Indeed, the CGRP has been involved in antioxidant activation, both in vivo and in vitro, other than in nociception $[18,19,24-26]$. In this scenario, we were not able to define which role is played exclusively by the CGRP-signaling blockage. However, we can speculate that even if both anti-CGRP antibodies and BoNT/A toxin treatments are effective in fairly similar populations, the antibodies failed to improve the antioxidant power of plasma, probably due to the blockage of CGRP antioxidant properties.

This evidence suggests that the CGRP system inhibition is indeed important for both enzymatic and non-enzymatic antioxidant power. However, the concentration of oxidation products was reduced, probably due to the improvement of migraine frequency or as a consequence of medication overuse discontinuation, as we previously suggested considering the BoNT/A treatment [15]. The CGRP system blockage also provides the same result, which could be independent of the chosen treatment, further validating the aforementioned hypothesis.. Our study has some limitations. First of all, the sample is relatively small, impeding sub-population analyses, as episodic versus chronic migraine, triptan versus NSAIDs overuser, or according to the administered antibody, to determine, for instance, if direct CGRP inhibition or its receptor could have different effects on oxidative stress.

Other comparative studies with similar populations are mandatory to further validate our hypotheses.

\section{Conclusions}

This study confirmed the safety and efficacy of the antibodies' anti-CGRP and its receptor in real-life, with patients characterized by a high-frequency, pharmacologically resistant migraine. The clinical improvement was also accompanied by a reduction in the oxidative stress biomarker, paving the way for the further understanding of migraine pathophysiology.

Additional studies will be necessary to understand the role of oxidative stress as a disease biomarker and how different treatments could affect the advanced oxidation products or the enzymatic and non-enzymatic antioxidant power of patients' plasma.

Author Contributions: Conceptualization and methodology, S.G. and C.D.L.; formal analysis and data curation, S.M. and F.B.; investigation, I.L., L.C. (Letizia Curto), S.G., F.B., and C.D.L.; resources, L.C. (Lucia Chico) and M.U.; writing-original draft preparation, C.D.L.; writing-review and editing, F.B. and M.P.; supervision, G.S.; project administration, S.G. All authors have read and agreed to the published version of the manuscript.

Funding: This research received no external funding.

Institutional Review Board Statement: This study was performed in accordance with the Declaration of Helsinki, and it was approved (protocol code: ID_14518, date 19 February 2019) by the local ethics committee (Comitato Etico Regionale per la Sperimentazione Clinica della Regione Toscanasezione: Area Vasta Nord Ovest, Stabilimento di Santa Chiara-Via Roma, 67-56126 Pisa). Informed consent was obtained from all subjects involved in the study.

Informed Consent Statement: Informed consent was obtained from all subjects involved in the study.

Data Availability Statement: The data presented in this study are available on request.

Conflicts of Interest: C.D.L. received an honorarium from Lilly. 


\section{References}

1. Headache Classification Committee of the International Headache Society (IHS). The International Classification of Headache Disorders, 3rd edition. Cephalalgia 2018, 38, 1-211. [CrossRef]

2. GBD 2019 Diseases and Injuries Collaborators. Global burden of 369 diseases and injuries in 204 countries and territories, 1990-2019: A systematic analysis for the Global Burden of Disease Study 2019. Lancet 2020, 396, 1204-1222. [CrossRef]

3. Lipton, R.B.; Silberstein, S.D. Episodic and Chronic Migraine Headache: Breaking Down Barriers to Optimal Treatment and Prevention. Headache 2015, 55 (Suppl. 2), 103-122. [CrossRef]

4. Edvinsson, L. Role of CGRP in Migraine. Handb. Exp. Pharmacol. 2019, 255, 121-130. [CrossRef]

5. Caronna, E.; Starling, A.J. Update on Calcitonin Gene-Related Peptide Antagonism in the Treatment of Migraine. Neurol. Clin. 2021, 39, 1-19. [CrossRef] [PubMed]

6. Sacco, S.; Lampl, C.; Van Den Brink, A.M.; Caponnetto, V.; Braschinsky, M.; Ducros, A.; Little, P.; Pozo-Rosich, P.; Reuter, U.; De La Torre, E.R.; et al. Burden and attitude to resistant and refractory migraine: A survey from the European Headache Federation with the endorsement of the European Migraine \& Headache Alliance. J. Headache Pain 2021, 22, 39. [CrossRef]

7. Ashina, M. Migraine. N. Engl. J. Med. 2020, 383, 1866-1876. [CrossRef] [PubMed]

8. Nowaczewska, M.; Straburzyński, M.; Meder, G.; Kaźmierczak, W. Changes in Cerebral Blood Flow after Erenumab Treatment in Good and Non-Responders-A Pilot Study of Migraine Patients. J. Clin. Med. 2021, 10, 2523. [CrossRef] [PubMed]

9. Bellei, E.; Bergamini, S.; Rustichelli, C.; Monari, E.; Porto, M.D.; Fiorini, A.; Tomasi, A.; Ferrari, A. Urinary Proteomics Reveals Promising Biomarkers in Menstrually Related and Post-Menopause Migraine. J. Clin. Med. 2021, 10, 1854. [CrossRef] [PubMed]

10. De Luca, C.; Gori, S.; Mazzucchi, S.; Dini, E.; Cafalli, M.; Siciliano, G.; Papa, M.; Baldacci, F. Supersaturation of VEP in Migraine without Aura Patients Treated with Topiramate: An Anatomo-Functional Biomarker of the Disease. J. Clin. Med. 2021, 10, 769. [CrossRef]

11. Ferroni, P.; Barbanti, P.; Spila, A.; Fratangeli, F.; Aurilia, C.; Fofi, L.; Egeo, G.; Guadagni, F. Circulating Biomarkers in Migraine: New Opportunities for Precision Medicine. Curr. Med. Chem. 2019, 26, 6191-6206. [CrossRef]

12. Pilati, L.; Battaglia, G.; Di Stefano, V.; Di Marco, S.; Torrente, A.; Raieli, V.; Firenze, A.; Salemi, G.; Brighina, F. Migraine and Sport in a Physically Active Population of Students: Results of a Cross-Sectional Study. Headache 2020, 60, 2330-2339. [CrossRef] [PubMed]

13. Visočnik, D.; Zaletel, M.; Žvan, B.; Zupan, M. Enhanced Hemodynamic and Clinical Response to $\alpha$ CGRP in Migraine Patients-A TCD Study. Front. Neurol. 2021, 12, 16. [CrossRef]

14. Lucchesi, C.; Cafalli, M.; Chico, L.; Gori, S.; Baldacci, F.; Gerfo, A.L.; Bonuccelli, U.; Siciliano, G. Evidences of Reduced Antioxidant Activity in Patients With Chronic Migraine and Medication-Overuse Headache. Headache 2015, 55, 984-991. [CrossRef] [PubMed]

15. Dini, E.; Mazzucchi, S.; De Luca, C.; Cafalli, M.; Chico, L.; Lo Gerfo, A.; Siciliano, G.; Bonuccelli, U.; Baldacci, F.; Gori, S. Plasma Levels of Oxidative Stress Markers, before and after BoNT/A Treatment, in Chronic Migraine. Toxins 2019, 11, 608. [CrossRef]

16. Cernuda-Morollón, E.; Ramón, C.; Martínez-Camblor, P.; Serrano-Pertierra, E.; Larrosa, D.; Pascual, J. OnabotulinumtoxinA decreases interictal CGRP plasma levels in patients with chronic migraine. Pain 2015, 156, 820-824. [CrossRef] [PubMed]

17. Gross, E.C.; Lisicki, M.; Fischer, D.; Sándor, P.S.; Schoenen, J. The metabolic face of migraine-From pathophysiology to treatment. Nat. Rev. Neurol. 2019, 15, 627-643. [CrossRef] [PubMed]

18. Nassini, R.; Materazzi, S.; Benemei, S.; Geppetti, P. The TRPA1 Channel in Inflammatory and Neuropathic Pain and Migraine. Rev. Physiol. Biochem. Pharmacol. 2014, 167, 1-43. [CrossRef]

19. Luo, H.; Wu, X.; Xian, X.; Wang, L.; Zhu, L.; Sun, H.; Yang, L.; Liu, W. Calcitonin gene-related peptide inhibits angiotensin II-induced NADPH oxidase-dependent ROS via the Src/STAT3 signalling pathway. J. Cell. Mol. Med. 2020, 24, 6426-6437. [CrossRef]

20. Sacco, S.; Braschinsky, M.; Ducros, A.; Lampl, C.; Little, P.; Brink, A.M.V.D.; Pozo-Rosich, P.; Reuter, U.; De La Torre, E.R.; Del Rio, M.S.; et al. European Headache Federation Consensus on the Definition of Resistant and Refractory Migraine: Developed with the Endorsement of the European Migraine \& Headache Alliance (EMHA). J. Headache Pain 2020, 21, 76 . [CrossRef]

21. Edvinsson, J.C.A.; Viganò, A.; Alekseeva, A.; Alieva, E.; Arruda, R.; De Luca, C.; D’Ettore, N.; Frattale, I.; Kurnukhina, M.; Macerola, N.; et al. The fifth cranial nerve in headaches. J. Headache Pain 2020, 21, 65. [CrossRef]

22. Aguggia, M. Allodynia and migraine. Neurol. Sci. 2012, 33 (Suppl. 1), 9-11. [CrossRef]

23. Di Stefano, V.; Ornello, R.; Gagliardo, A.; Torrente, A.; Illuminato, E.; Caponnetto, V.; Frattale, I.; Golini, R.; Di Felice, C.; Graziano, F.; et al. Social Distancing in Chronic Migraine during the COVID-19 Outbreak: Results from a Multicenter Observational Study. Nutrients 2021, 13, 1361. [CrossRef]

24. Geyik, S.; Altunısık, E.; Neyal, A.M.; Taysi, S. Oxidative stress and DNA damage in patients with migraine. J. Headache Pain 2016, 17, 10. [CrossRef]

25. Bulboacă, A.E.; Stănescu, I.C.; Bolboacă, S.D.; Bulboacă, A.C.; Bodizs, G.I.; Nicula, C.A. Retinal Nerve Fiber Layer Thickness and Oxidative Stress Parameters in Migraine Patients without Aura: A Pilot Study. Antioxidants 2020, 9, 494. [CrossRef]

26. Liu, Y.; Zhang, S.; Xue, J.; Wei, Z.; Ao, P.; Shen, B.; Ding, L. CGRP Reduces Apoptosis of DRG Cells Induced by High-Glucose Oxidative Stress Injury through PI3K/AKT Induction of Heme Oxygenase-1 and Nrf-2 Expression. Oxidative Med. Cell. Longev. 2019, 2019, 2053149. [CrossRef] 